\title{
Right atrial pacing in patients with sinus node dysfunction results from an observational cohort study
}

\author{
Márcio Galindo Kiuchi ${ }^{1 *}$, ShaojieChen ${ }^{2,3}$, Gustavo Ramalho e Silva ${ }^{1}$, Luis Marcelo Rodrigues Paz ${ }^{1}$ and Gladyston Luiz Lima Souto ${ }^{1}$ \\ ${ }^{1}$ Cardiac Surgery Division, Department of Medicine, Hospital e Clínica São Gonçalo, São Gonçalo, RJ, Brazil \\ ${ }^{2}$ Department of Cardiology, Shanghai First People's Hospital, Shanghai Jiao Tong University School of Medicine, Shanghai, China \\ ${ }^{3}$ Fellowship of the European Heart Rhythm Association/European Society of Cardiology, Department of Cardiology, Elisabethinen University Teaching Hospital \\ Linz, Linz, Austria
}

\begin{abstract}
Purpose: This study examined patients who had a DDDR pacemaker and were exposed to different degrees of atrial and ventricular pacing. We aimed to determine the relationships between baseline features and percentages of atrial pacing (\%AP), and ventricular pacing (\%VP) in the first month post implant and subsequent onset of atrial fibrillation (AF) (primary outcomes). We also aimed to determine the relationships between time-dependent changes in \%AP and \%VP and AF recurrence (secondary outcomes).

Methods: Three hundred six patients were evaluated every 6 months up to 3 years of follow-up. The \%AP and \%VP were assessed at each follow-up visit.

Results: The mean time to the first episode of AF ( $\mathrm{n}=227)$ was $15.3 \pm 10.7$ months. At the end of 3 years of follow-up, $74.2 \%$ patients developed AF events. At baseline, the mean \%AP was significantly higher inpatients who subsequently developed AF than in those who did not $(68.5 \% \pm 19.7 \%$ versus $33.3 \% \pm 23.2 \%$, $\mathrm{P}<0.0001)$. Similarly, mean $\%$ VP was significantly higher in patients who developed AF than in those without AF events $(25.8 \% \pm 14.5 \%$ versus $14.4 \% \pm 11.9 \%$, $\mathrm{P}<0.0001$ ). Kaplan-Meier survival analysis demonstrated a significant increased risk of AF events in patients who subsequently developed AF compared with those who did not (log-rank test, $\mathrm{P}<0.0001)$
\end{abstract}

Conclusions: Our study shows an association between \%AP and \%VP at baseline and time to appearance of the first AF episode after pacemaker implantation. During 3 years of follow-up, we also observed a significantly increased risk of AF events in the high \%AP and high \%VP groups at baseline.

List of Abbreviations: AF: atrial fibrillation, AMS: auto mode switch, AP: atrial pacing, \%AP: percentage of atrial pacing, AT: atrial tachycardia, AUC: area under the curve, AV: atrioventricular, LAD: left atrial diameter, LVEF: left ventricular ejection fraction, ROC: receiver operator characteristic, SND: sinus node disease, SSS: sick sinus syndrome, VP: ventricular pacing, \%VP: percentage of ventricular pacing

\section{Introduction}

Sinus node dysfunction is characterized by one or more of the following electrocardiographic manifestations: sinus bradycardia, sinus arrest, sino-atrial block, and supraventricular tachycardia alternating with sinus bradycardia and asystole (Brady-tachycardia syndrome) [1]. The presence of palpitations, dizziness, pre-syncope, or syncope associated with these electrocardiographic changes defines sinus node disease (SND). SND occurs more frequently in women than in men, resulting in a high morbidity between 60 and 69 years $[2,3]$. The most common form of SND has no clear etiology and is considered idiopathic or primary [4,5]. Paroxysmal atrial fibrillation (AF) is common in patients with sick sinus syndrome (SSS) and pacemakers, and leads to morbidity and an increased risk of stroke or death [6].

The recent DANPACE studyenrolled patients with SSS with no previous history of AF who received DDDR pacemakers [7,8], and aimed to determine the effect of atrial pacing on the occurrence of AF. This study did not find an association between increased atrial pacing and development of AF $[7,8]$.
The present study examined patients who had a DDDR pacemaker and were exposed to different degrees of atrial pacing (AP) and ventricular pacing (VP). We aimed to determine the relationships between baseline features and percentages of atrial pacing (\%AP) and ventricular pacing (\%VP) in the first month postimplant and subsequent onset of AF (primary outcomes).Moreover, we aimed to determine the relationships between time-dependent changes in \%AP and $\% \mathrm{VP}$ and $\mathrm{AF}$ recurrence (secondary outcomes), thus allowing to treat the disease at earlier stages and change the natural course of this and its possible complications.

\section{Methods}

\section{Study design}

The present observational, prospective study was conducted at the Department of Cardiac Pacing and Cardiac Surgery of the Hospital e Clínica São Gonçalo. A cohort of patients received standard therapy

Correspondence to: Márcio Galindo Kiuchi, Rua Cel, Moreira César, 138 Centro, São Gonçalo - Rio de Janeiro - Brazil. ZIP-CODE: 24440-400, Tel/Fax: +55 (21) 26047744; E-mail: marciokiuchi@gmail.com

Key words: sinus node disease, sick sinus syndrome, pacemaker, atrial fibrillation, pacing, left atrial diameter

Received: September 24, 2016; Accepted: October 04, 2016; Published: October 07,2016 
for treatment of SND and DDDR pacemaker implantation. Followup was 3 years after the implantation procedure. The study inclusion criteria were as follows: (i) patients did not have electrocardiogramdocumented AF or a previous history of paroxysmal AF; (ii) patients provided documentation of no cardiac ischemia before pacemaker implantation as proven by a myocardial scintigraphy at rest and during stress, a cardiac magnetic resonance imaging at rest and during stress, or pharmacological stress echocardiography; (iii) patients had a left ventricular ejection fraction (LVEF) $\geq 50 \%$ as measured by echocardiography; (iv) tests showing that the patients had SND (symptomatic bradycardia; documented sino-atrial block or sinus arrest with pauses $>3 \mathrm{~s}$ or sinus bradycardia $<40 \mathrm{bpm}$ for $>1$ min while awake) before pacemaker implantation; and (v) tests showing that patients had no second- or third-degree atrioventricular (AV) block, and a PR interval $<240 \mathrm{~ms}$ before pacemaker implantation.

Exclusion criteria were as follows: (i) ischemic heart disease; (ii) an LVEF <50\%; (iii) heart valvar disease that may lead to AF; and (iv) symptoms suggestive of AF.

Enrolment of patients started in January 2009 and was terminated in September 2012. Patients were followed up until September 2015, and they were identified at our offices. The study was conducted in accordance with the Helsinki Declaration and was approved by the Ethics Committee of our hospital. All of the patients gave written informed consent before inclusion.

\section{Implantation and programing of pacemakers}

As a routine practice in our department, bipolar leads were implanted in the appendage of the right atrium and in the high septal region of the right ventricle. DDDR pacemakers from St. Jude Medical (St. Jude Medical, St. Paul, Minnesota, USA) and Medtronic (Medtronic, Palo Alto, CA, USA) were used. The rate adaptive function was activated in all of the pacemakers and programed with a lower rate of $60 \mathrm{bpm}$ and an upper rate of $120 \mathrm{bpm}$ In all of the pacemakers, we programed the paced atrioventricular interval to $140-220 \mathrm{~ms}$ and turned on the AV delay management algorithm that automatically searches for intrinsic conduction to prevent unnecessary right ventricular pacing. The maximum tracking rate was individualized and the auto mode switch (AMS) function was activated. AMS occurred when the atrial rate exceeded $170-180 \mathrm{bpm}$ for a specific number of beats or period of time. The atrial tachycardia/atrial fibrillation (AT/ AF) diagnostic suite provided detailed historical data, allowing us to identify and evaluate therapy for improved management of patients. Atrial sensitivity was programed to $0.5 \mathrm{mV}$.

\section{Definition of atrial fibrillation}

AF was defined as at least one episode of atrial irregular activity recorded by the atrial channel lasting $\geq 30 \mathrm{~s}$.

\section{Patients' follow-up}

Patients were evaluated 15 days after pacemaker implantation to assess the pocket, the site of the surgical incision, and to adjust the programing of the pacemaker. Fifteen days later, the patients returned for reassessment (1 month after pacemaker implantation). Data were obtained from the pacemaker at 1 month postimplant. Thereafter, patients were assessed every 6 months up to 3 years of follow-up. At each follow-up visit, we obtained a record (stored on a USB stick and then transferred to a computer) of the pacemaker memory data that had accumulated since the previous resetting of the memory. The \% AP and \%VP at each follow-up were calculated by the pacemaker using the number of paced and sensed beats. The occurrence and duration of AMS events were recorded. The onset of the first AF episode was also registered in each patient's data record. Time to AF onset was defined as the number of days from baseline to the first recorded episode of AF lasting $\geq 30$ s. Patients were censored due to death, loss to follow-up, or 3 years post-implant. According to our standard of care, all of the patients underwent echocardiography at baseline and annually after pacemaker implantation.

\section{Statistical analysis}

All enrolled patients were included in the analyses. Variables were compared between two groups using analysis of variance (ANOVA) for continuous measures and the $\chi^{2}$ test or Fisher's exact test for categorical variables. The Kaplan-Meier method was used to describe the freedom from AF events over time, and differences between groups were assessed by the log-rank test. Baseline covariates and disease characteristics related to AF recurrence $(\mathrm{P}<0.1)$ were included in the Cox proportional hazards regression model, along with baseline \%AP, $\% \mathrm{VP}$, and left atrial diameter (LAD). Forward selection was used to reduce the number of covariates. Receiver operator characteristic (ROC) curves were used to test the effectiveness of \%AP and \%VP at baseline in predicting AF events, and the area under the curve (AUC) was derived from the ROC curve. All statistical tests were two-tailed, and a $P$ value of $<0.05$ was considered to indicate statistical significance. All statistical analyses were performed using SPSS v 18.0.

\section{Results}

\section{Patients}

We screened 391 patients who received a DDD pacemaker to treat SND for preliminary inclusion (Figure 1). Among these, 85 patients were excluded because they fulfilled the following exclusion criteria: ischemic heart disease $(n=21)$; an LVEF $<50 \%(n=33)$; and symptoms suggestive of $A F(n=31)$. The remaining 306 patients who adhered to the inclusion criteria were finally enrolled in the study (group 1: patients who did not develop AF, $n=79$; group 2: patients who developed AF, $\mathrm{n}=227$ ). The baseline characteristics of patients in both groups are shown in Table 1.

\section{The \%AP and \%VP at baseline compared with the probability of AF}

At baseline, mean \%AP was significantly higher in patients who subsequently developed AF compared with those who did not $(33.3 \%$ $\pm 23.2 \%$ versus $68.5 \% \pm 19.7 \%, \mathrm{P}<0.0001)$. Similarly, mean $\%$ VP was significantly higher in patients who developed AF compared with those who did not $(25.8 \% \pm 14.5 \%$ versus $14.4 \% \pm 11.9 \%, \mathrm{P}<0.0001)$.

\section{AF events}

The mean time to the first episode of $\mathrm{AF}(\mathrm{n}=227)$ was $15.3 \pm 10.7$ months. At the end of 3 years of follow-up, $25.8 \%$ of patients did not have AF episodes, while $74.2 \%$ of patients developed AF events.

ROC analysis showed that the \%AP and \%VP at baseline were feasible and effective predictors for the long-term risk of AF episodes (AUC: $0.8828,95 \%$ confidence interval: $0.8267-0.9390, \mathrm{P}<0.0001$; AUC: $0.7484,95 \%$ confidence interval: $0.6836-0.8131, \mathrm{P}<0.0001$, respectively). The potential cut-off points of $\% \mathrm{AP}$ and $\% \mathrm{VP}$ derived from ROC analysis were $45.5 \%$ (sensitivity: $91.63 \%$; specificity: $81.01 \%$ ) and $18.5 \%$ (sensitivity: $68.72 \%$; specificity: $72.15 \%$ ), respectively (Figure $2 \mathrm{~A}$ and $2 \mathrm{~B}$ ). Using these thresholds, Kaplan-Meier survival analysis 


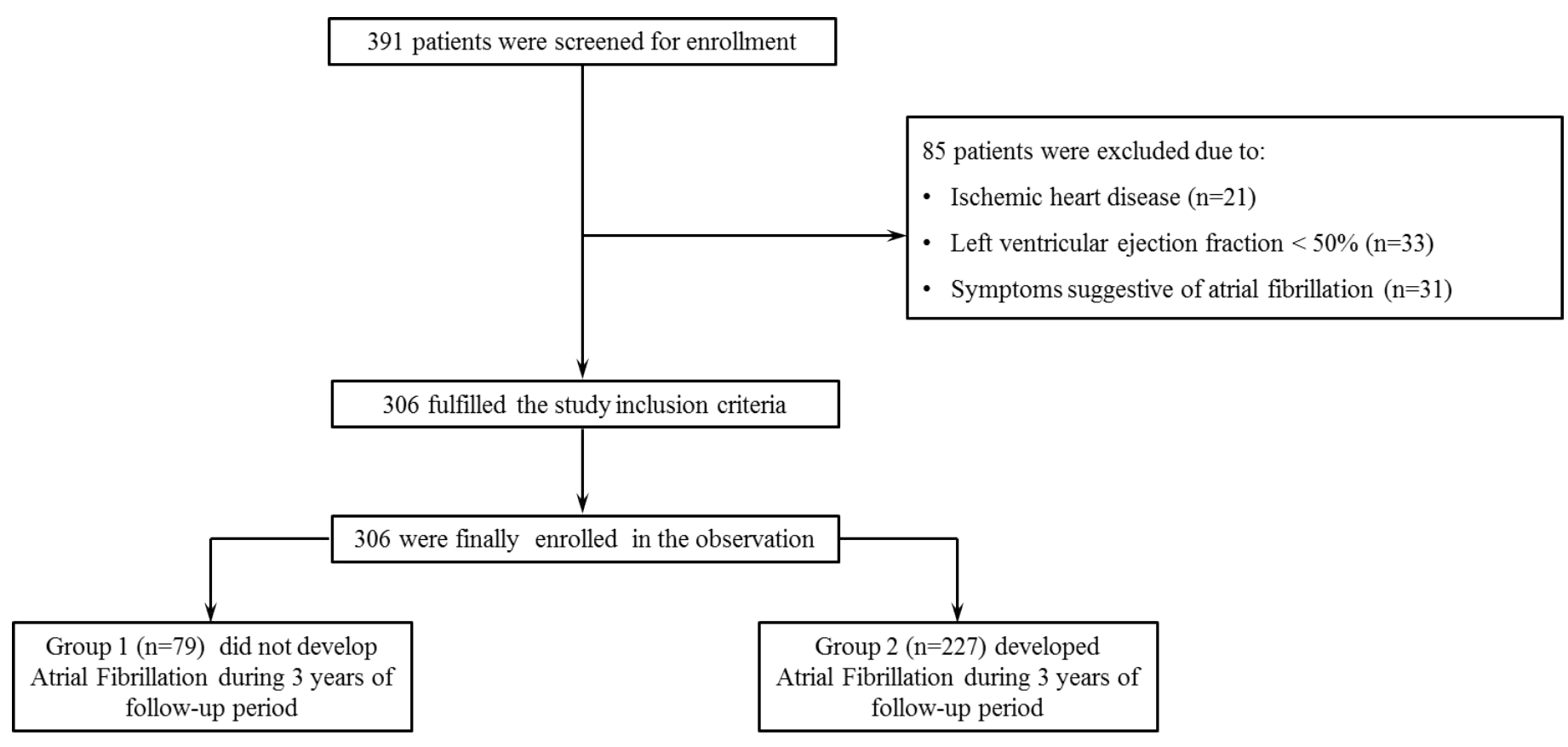

Figure 1. Flow chart of the study. Enrolment, stratification, and follow-up of the patients are shown.

Table 1. Baseline features of included patients.

\begin{tabular}{|c|c|c|c|c|}
\hline Variables & Overall n=306 & $\begin{array}{c}\text { Group } 1 \\
n=79\end{array}$ & Group $2 n=227$ & Pvalue \\
\hline Age (years) & $62.3 \pm 13.2$ & $61.8 \pm 8.8$ & $65.3 \pm 12.4$ & 0.0194 \\
\hline Sex, male, $n(\%)$ & $173(56.5)$ & $42(53.2)$ & $131(57.7)$ & 0.5116 \\
\hline Hypertension, n (\%) & $139(45.4)$ & $16(20.3)$ & $123(54.2)$ & $<0.0001$ \\
\hline $\mathrm{CAD}^{\mathrm{a}}, \mathrm{n}(\%)$ & $31(10.1)$ & $8(10.1)$ & $23(10.1)$ & 1.000 \\
\hline Diabetes mellitus, n (\%) & $132(43.1)$ & $22(27.8)$ & $110(48.5)$ & 0.0038 \\
\hline ACEI or ARB, n (\%) & $125(40.8)$ & $14(17.7)$ & $111(48.9)$ & $<0.0001$ \\
\hline Diuretics, $\mathrm{n}(\%)$ & $45(14.7)$ & $3(3.8)$ & $42(18.5)$ & 0.0008 \\
\hline $\mathrm{Ca}^{++}$channel blockers, $\mathrm{n}(\%)$ & $46(15.0)$ & $2(2.5)$ & $44(19.4)$ & $<0.0001$ \\
\hline Beta-blockers, n (\%) & $29(9.5)$ & $7(8.9)$ & $22(9.7)$ & 1.0000 \\
\hline $\mathrm{AP}(\%)$ & $59.4 \pm 25.7$ & $33.3 \pm 23.3$ & $68.5 \pm 19.7$ & $<0.0001$ \\
\hline VP (\%) & $23.4 \pm 17.9$ & $14.4 \pm 11.9$ & $26.6 \pm 18.6$ & $<0.0001$ \\
\hline $\mathrm{LAD}(\mathrm{cm})$ & $3.9 \pm 0.4$ & $3.6 \pm 0.2$ & $4.0 \pm 0.4$ & 0.6161 \\
\hline LVEF (\%) & $64.2 \pm 7.3$ & $63.8 \pm 7.4$ & $64.4 \pm 7.3$ & 0.9996 \\
\hline
\end{tabular}

ACEI: angiotensin-converting enzyme inhibitor; AP: atrial pacing; ARB: angiotensin-receptor blocker; CAD: coronary artery disease; LAD: left atrial diameter; LVEF: left ventricular ejection fraction; VP: ventricular pacing.

aPatients with coronary artery disease had undergone coronary angiography or computed tomography angiography of coronary arteries at some time in their life. This indicated that they had some degree of obstruction in the coronary arteries, but this did not cause ischemia.

demonstrated a significant increased risk of AF events in the high \%AP group ( $>45.5 \%)$ and in the high \%VP group ( $>18.5 \%$ ) (both $\mathrm{P}<0.0001$, log-rank test, Figure $3 \mathrm{~A}$ and $3 \mathrm{~B}$, respectively).

\section{The \%AP and \%VP, left atrial diameter, and LVEF during follow-up}

Throughout follow-up, we did not observe changes in \%AP \%VP, and LVEF in both groups. However, LAD showed reduction in group 1 in comparison to group 2 at specific time points (Table 2).

\section{Adjustment for several possible confounders known to be associated with AF}

As shown in Table 3, age, hypertension, diabetes, use of angiotensinconverting enzyme inhibitor/angiotensin-receptor blocker (ACEI/ $\mathrm{ARB})$, diuretics, calcium channel blockers (CCB), \%AP, and \%VP were significantly different (defined as $\mathrm{P}<0.1$ ) between the groups. We included these variables plus LAD and LVEF (they are both outcomerelevant measurements) in the Cox multivariate logistic regression model by the forward stepwise (Wald) method. Male sex, hypertension, $\% \mathrm{AP}, \% \mathrm{VP}$, and LAD were risk factors of development of AF (Table 4).

\section{Discussion}

In the present study, we found associations of \%AP and \%VP at baseline, as well as male sex, hypertension, and higher left atrial diameter with the time to appearance of the first AF episode after pacemaker implantation. We also found that by using the thresholds from the ROC curve, Kaplan-Meier survival analysis showed a significant increased risk of AF events in the high \%AP and high \%VP groups.

Elkayam et al. [9] reported that AP and VP in patients with SND 
(A)

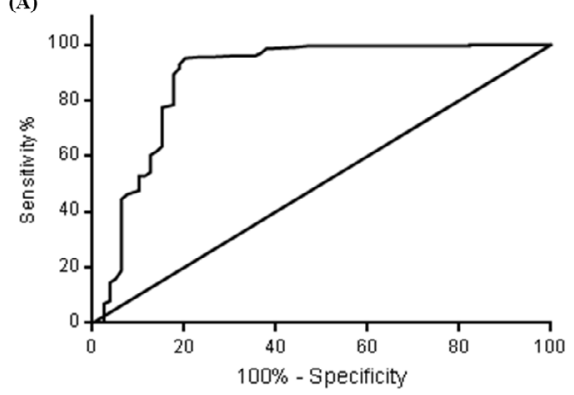

Area Under Curve 0.8828. 95\% CI: 0.8267-0.9390. P $<0.000$

(B)

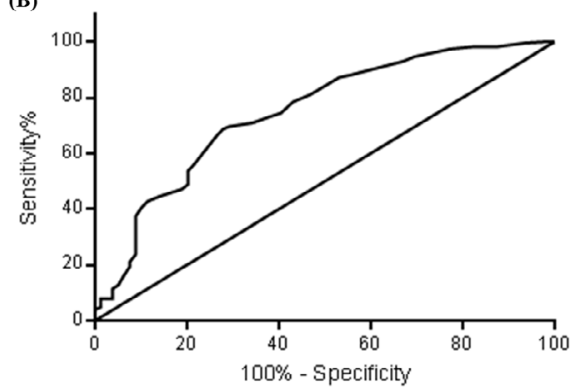

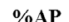

38.50

40.50

42.50

43.50

44.50

45.50

46.50

47.50

$\begin{array}{cc}\text { Sensitivity\% } & \text { Specificity\% } \\ 95.59 & 74.68 \\ 95.59 & 75.95 \\ 95.59 & 77.22 \\ 95.15 & 79.75 \\ 92.95 & 81.01 \\ \mathbf{9 1 . 6 3} & \mathbf{8 1 . 0 1} \\ 89.43 & 82.28 \\ 87.22 & 82.28\end{array}$

\%VP

14.50

15.50

16.50

17.50

18.50

19.50

20.50

21.50

$\begin{array}{cc}\text { Sensitivity\% } & \text { Specificity\% } \\ 74.45 & 59.49 \\ 73.13 & 62.03 \\ 70.93 & 65.82 \\ 69.60 & 70.89 \\ \mathbf{6 8 . 7 2} & 72.15 \\ 66.52 & 73.42 \\ 58.59 & 77.22 \\ 55.95 & 78.48\end{array}$

Area Under Curve 0.7484. 95\% CI: 0.6836-0.8131. P $<0.0001$
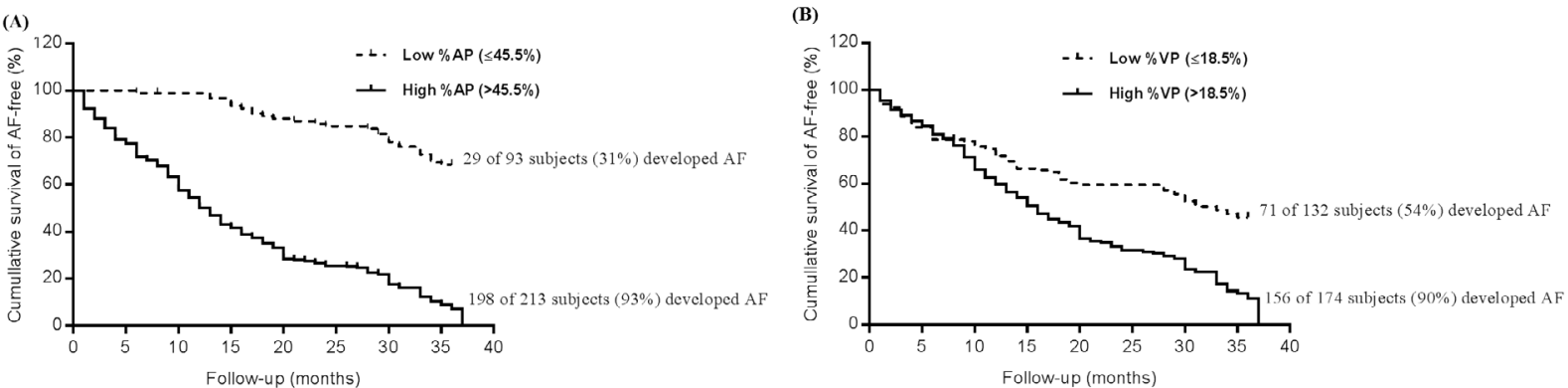

Figure 3. Kaplan-Meier analysis for AF events in (A) low and high \%AP, and (B) in low and high \%VP at baseline (both P<0.0001). AF: atrial fibrillation; AP: atrial pacing; VP: ventricular pacing

were predisposing factors for development of AF. However, the DANPACE trial showed that in 396 patients who received DDDR pacemakers because of SSS, 72\% developed AF as indicated by AMS episodes at some point during follow-up [8]. This trial also showed no association between \%AP and development of AF [8].

We found that the mean time for the first AF episode after pacemaker implantation was $15.3 \pm 10.7$ months, and among 306 patients, $74 \%$ developed AF. The risk of stroke in patients with AF can be evaluated by using the CHADS2 and the CHA2DS2-VASc score system. Svendsen et al. hypothesized that these risk scores and their individual components can also be applied to patients paced for SSS to evaluate the risk of stroke and death [10]. They showed that CHADS2 and CHA2DS2-VASc scores were associated with an increased risk of stroke and death in patients paced for SSS, irrespective of the presence of AF. In our study, the mean \% AP and \%VP in patients who did not develop AF were lower than those in patients who showed AF events during the follow-up period. In patients without AF, the \%AP was $34 \%$ and the $\% \mathrm{VP}$ was $14 \%$ at the $36^{\text {th }}$ month of follow-up. In patients that developed AF, the \%AP was $72 \%$ and the $\% \mathrm{VP}$ was $25 \%$ at the $36^{\text {th }}$ month of follow-up. The variation $(\Delta)$ between these two groups was $38 \%$ for $\% \mathrm{AP}(72 \%-34 \%)$ and $9 \%$ for $\% \mathrm{VP}(25 \%-14 \%)$ at the $36^{\text {th }}$ month of follow-up. Furthermore, ROC analysis showed that \%AP and $\% \mathrm{VP}$ were feasible and effective predictors for long-term risk of AF episodes. The potential cut-off points of $\% \mathrm{AP}$ and \%VP derived from ROC analysis were $45.5 \%$ and $18.5 \%$, respectively. These thresholds can be important because they may allow identification of potential individuals who will develop AF. This enables patients to be more closely and routinely followed up. AP is considered potentially proarrhythmogenic by some scientists, while others have focused on atrial overdrive pacing strategies for preventing AF. Results from studies on overdrive pacing have not shown a convincing benefit in prevention of $\mathrm{AF}$, and these trials only had modest sample sizes $[11,12]$. The ASSERT trial with 2580 patients did not demonstrate any benefit of continuous atrial overdrive pacing [13].

Pacing in the right atrium may induce atrial dyssynchrony and subsequent AF [14]. The percentage of atrial pacing can lead to hemodynamic alterations, and changes in the shape and velocity of intra-atrial, interatrial, and atrioventricular conduction, as well as 
Table 2. \%AP and \%VP during 3 years of follow up.

\begin{tabular}{|c|c|c|c|c|c|c|c|c|}
\hline$\% \mathbf{A P}$ & Baseline & 6 months & 12 months & 18 months & 24 months & 30 months & 36 months & All P-value \\
\hline Overall & $59.42 \pm 25.72$ & $60.38 \pm 25.73$ & $60.84 \pm 25.80$ & $61.21 \pm 26.06$ & $61.74 \pm 26.13$ & $62.04 \pm 26.31$ & $62.53 \pm 26.31$ & 0.8041 \\
\hline Group 1 & $33.33 \pm 23.15$ & $33.86 \pm 23.13$ & $33.87 \pm 22.94$ & $34.01 \pm 23.18$ & $34.51 \pm 23.5$ & $34.14 \pm 23.62$ & $34.34 \pm 23.60$ & $>0.9999$ \\
\hline Group 2 & $68.51 \pm 19.67$ & $69.61 \pm 19.43$ & $70.23 \pm 19.33$ & $70.68 \pm 19.56$ & $71.22 \pm 19.54$ & $71.74 \pm 19.36$ & $72.34 \pm 19.16$ & 0.4066 \\
\hline$\Delta$ group $v s$. group 2 & 35.18 & 35.75 & 36.36 & 36.67 & 36.71 & 37.61 & 38.00 & $<0.0001$ \\
\hline$\% \mathrm{VP}$ & Baseline & 6 months & 12 months & 18 months & 24 months & 30 months & 36 months & All P-value \\
\hline Overall & $23.43 \pm 17.92$ & $23.04 \pm 14.84$ & $23.07 \pm 14.65$ & $22.87 \pm 14.36$ & $22.69 \pm 14.08$ & $22.30 \pm 14.07$ & $22.41 \pm 14.03$ & 0.9719 \\
\hline Group 1 & $14.41 \pm 11.87$ & $14.30 \pm 11.64$ & $14.30 \pm 11.33$ & $14.11 \pm 10.86$ & $14.16 \pm 10.56$ & $14.10 \pm 10.46$ & $14.51 \pm 10.70$ & $>0.9999$ \\
\hline Group 2 & $26.57 \pm 18.61$ & $26.08 \pm 14.65$ & $26.11 \pm 14.47$ & $25.92 \pm 14.19$ & $25.65 \pm 13.96$ & $25.15 \pm 14.06$ & $25.16 \pm 14.03$ & 0.9479 \\
\hline$\Delta$ group $v s$. group 2 & 12.16 & 11.78 & 11.81 & 11.80 & 11.49 & 11.05 & 10.66 & $<0.0001$ \\
\hline $\operatorname{LAD}(\mathbf{c m})$ & Baseline & \multicolumn{2}{|c|}{12 months } & \multicolumn{2}{|c|}{24 months } & \multicolumn{2}{|c|}{36 months } & All P-value \\
\hline Overall & $3.86 \pm 0.42$ & \multicolumn{2}{|c|}{$4.05 \pm 2.51$} & \multicolumn{2}{|c|}{$3.95 \pm 0.45$} & \multicolumn{2}{|c|}{$4.08 \pm 1.83$} & 0.3023 \\
\hline Group 1 & $3.59 \pm 0.22$ & \multicolumn{2}{|c|}{$3.59 \pm 0.22$} & \multicolumn{2}{|c|}{$3.60 \pm 0.22$} & \multicolumn{2}{|c|}{$3.61 \pm 0.22$} & 0.8993 \\
\hline Group 2 & $3.96 \pm 0.43$ & \multicolumn{2}{|c|}{$4.22 \pm 2.90$} & \multicolumn{2}{|c|}{$4.07 \pm 0.45$} & \multicolumn{2}{|c|}{$4.24 \pm 2.10$} & 0.3068 \\
\hline group $1 v s$. group 2 & 0.3699 & \multicolumn{2}{|c|}{$0.6243 *$} & \multicolumn{2}{|c|}{0.46910} & \multicolumn{2}{|c|}{$0.6256^{*}$} & $*<0.05$ \\
\hline $\operatorname{LEVF}(\%)$ & Baseline & \multicolumn{2}{|c|}{12 months } & \multicolumn{2}{|c|}{24 months } & \multicolumn{2}{|c|}{36 months } & All P-value \\
\hline Overall & $64.22 \pm 7.34$ & \multicolumn{2}{|c|}{$64.19 \pm 7.33$} & \multicolumn{2}{|c|}{$64.93 \pm 13.95$} & \multicolumn{2}{|c|}{$64.28 \pm 7.35$} & 0.7293 \\
\hline Group 1 & $63.75 \pm 7.41$ & \multicolumn{2}{|c|}{$63.77 \pm 7.41$} & \multicolumn{2}{|c|}{$66.47 \pm 24.50$} & \multicolumn{2}{|c|}{$64.04 \pm 7.25$} & 0.5430 \\
\hline Group 2 & $64.38 \pm 7.32$ & \multicolumn{2}{|c|}{$64.33 \pm 7.31$} & \multicolumn{2}{|c|}{$64.4 \pm 7.32$} & \multicolumn{2}{|c|}{$64.36 \pm 7.39$} & 0.9997 \\
\hline group 1 vs. group 2 & 0.6364 & \multicolumn{2}{|c|}{0.5582} & \multicolumn{2}{|c|}{2.070} & \multicolumn{2}{|c|}{0.3249} & 0.7014 \\
\hline
\end{tabular}

AP: atrial pacing, LAD: left atrial diameter, LVEP: left ventricular ejection frction, VP: ventricular pacing

Table 3. Cox proportional hazards model to examine the associations between variables and $\mathrm{AF}$ events.

\begin{tabular}{|l|c|c|c|}
\hline Variables & OR & $\mathbf{9 5 \% C I}$ & Pvalue \\
\hline Age & 0.995 & $0.981-1.009$ & 0.469 \\
\hline Male sex & 1.363 & $1.019-1.822$ & 0.037 \\
\hline Hypertension & 2.062 & $1.018-4.178$ & 0.044 \\
\hline Diabetes mellitus & 0.949 & $0.705-1.277$ & 0.729 \\
\hline Coronary artery disease & 0.782 & $0.320-1.910$ & 0.590 \\
\hline \%Atrial pacing & 1.030 & $1.022-1.038$ & $<0.001$ \\
\hline \%Ventricular pacing & 1.021 & $1.012-1.029$ & $<0.001$ \\
\hline Left ventricular ejection fraction & 0.993 & $0.974-1.013$ & 0.501 \\
\hline Left atrial diameter & 11.110 & $6.209-19.879$ & $<0.001$ \\
\hline ACEI/ARB & 0.911 & $0.477-1.738$ & 0.776 \\
\hline Diuretic & 0.954 & $0.646-1.411$ & 0.815 \\
\hline Calcium channel blocker & 1.093 & $0.741-1.613$ & 0.654 \\
\hline B-blocker & 0.994 & $0.393-2.517$ & 0.991 \\
\hline
\end{tabular}

ACEI: angiotensin-converting enzyme inhibitor; AF: atrial fibrillation; ARB: angiotensinreceptor blocker; OR: odds ratio; 95\% CI: 95\% confidence interval.

Table 4. Cox proportional hazards model by the forward stepwise (Wald) method to examine the associations between variables and $\mathrm{AF}$ events.

\begin{tabular}{|l|c|c|c|}
\hline Variables & OR & $\mathbf{9 5 \% C I}$ & Pvalue \\
\hline Male sex & 1.341 & $1.016-1.770$ & 0.038 \\
\hline Hypertension & 1.941 & $1.458-2.584$ & $<0.0001$ \\
\hline \%Atrial pacing & 1.028 & $1.021-1.035$ & $<0.001$ \\
\hline \%Ventricular pacing & 1.021 & $1.013-1.029$ & $<0.001$ \\
\hline Left atrial diameter & 11.059 & $6.550-18.673$ & $<0.001$ \\
\hline
\end{tabular}

AF: atrial fibrillation; OR: odds ratio; 95\%CI: 95\% confidence interval.

alter cellular properties. An association between \%AP and AF may be possible because of differences among baseline variables. Theoretically, a lower intrinsic heart rate may indicate a greater severity of SSS and thus be associated with a higher \%AP and a risk of developing AF, without implications of a causal connection. The rate response to be associated with a higher degree of AP, it also theoretically possible, that this feature is another contributing factor. Because all of the patients in our study had this feature turned on, we are not able to confirm this possibility. Pacing strategies targeting the intra- and interatrial conduction tracts $[15,16]$ and algorithms aimed at minimizing atrial pacing should be further examined.

The mechanism for the increased incidence of AF with VP remains unclear, but hemodynamics are thought to be involved. Right VP leads to left ventricular remodeling, increases mitral regurgitation, and modestly reduces ejection fraction [17]. Furthermore, changing the relationship between atrial and ventricular timing, as can occur with VP, increases atrial pressure and causes stretch-related changes. This may increase the incidence of AF [18].

In our study, male sex, hypertension, and a higher LAD appeared to be associated with onset of $\mathrm{AF}$, despite using antihypertensive drugs, including ACEI/ARB. A higher LAD might be due to increased intracavitary pressure and possible scar tissue areas on the left atrium. Furthermore, the state of sympathetic overactivity present in hypertension may contribute to triggering factors involved in AF.

\section{Limitations}

A limitation of this study is that patients who were included in the study could have presented with an episode of asymptomatic AF. However, this was unable to be determined because they did not have an implantable looper.

The occurrence of AF may sometimes lead to under-sensing in the atrium and thus inappropriate AP. The use of beta-blockers and the onset of antiarrhythmic drugs after the first AF event may inhibit the sinus node and thus cause an increase in \%AP. However, we were unable to satisfactorily adjust for beta-blocker or antiarrhythmic drug use because of the heterogeneity of drugs and doses used, and their wide variation of effects in patients. Some comorbid conditions, such as hypertension, diabetes, and a high degree of sinus dysfunction, more common in one of the groups, led to a higher percentage of atrial pacing and probably to onset of AF. Furthermore, the causality of atrial pacing and development of AF was not able to be determined in this study.

\section{Conclusion}

The present study shows associations of $\% \mathrm{AP}$ and $\% \mathrm{VP}$ at baseline, 
male sex, hypertension, and higher left atrial diameter with the time to appearance of the first $\mathrm{AF}$ episode after pacemaker implantation. We also observed a significant increased risk of AF events in the high \%AP and high \%VP groups during 3 years of follow-up.

\section{Compliance with ethical standards}

All procedures performed in studies involving human participants were in accordance with the ethical standards of the institutional and/ or national research committee and with the 1964 Helsinki declaration and its later amendments or comparable ethical standards. Informed consent was obtained from all individual participants included in the study

\section{Funding}

The study was sponsored by health insurance plans of the state of Rio de Janeiro and Pacemed.

\section{Conflict of interest}

The authors declare that they have no conflict of interest.

\section{Acknowledgements}

The authors thank all the participants in this study and Pacemed for the technical support. The study was sponsored by health insurance plans of the state of Rio de Janeiro and Pacemed.

\section{References}

1. Ferrer MI (1968) The sick sinus syndrome in atrial disease. JAMA 206: 645-646. [Crossref]

2. Shaw DB, Holman RR, Gowers JI (1980) Survival in sinoatrial disorder (sick-sinus syndrome). Br Med J 280: 139-141. [Crossref]

3. de Marneffe M, Gregoire JM, Waterschoot P, Kestemont MP (1993) The sinus node function: normal and pathological. Eur Heart $J$ 14: 649-654. [Crossref]

4. Shaw DB (1976) The etiology of sino-atrial disorder (Sick Sinus Syndrome). Am Heart J 92: 539-540. [Crossref]

5. Nieminski KE, Kay RH, Rubin DA (1984) Current concepts and management of the sick sinus syndrome. Heart Lung 13: 675-681. [Crossref]

6. Glotzer TV, Hellkamp AS, Zimmerman J, Sweeney MO, Yee R, et al. (2003) MOST Investigators (2003). Atrial high rate episodes detected by pacemaker diagnostics predict death and stroke: report of the Atrial Diagnostics Ancillary Study of the MOde Selection Trial (MOST). Circulation 107: 1614-1619.

7. Nielsen JC, Thomsen PE, Højberg S, Møller M, Vesterlund T, et al. (2011) A comparison of single-lead atrial pacing with dual-chamber pacing in sick sinus syndrome. Eur Heart J 32: 686-696. [Crossref]

8. Hjortshøj S, Riahi S, Nielsen JC, Skjøth F, Lundbye-Christensen S, et al. (2014) DANPACE Investigators. Does atrial pacing lead to atrial fibrillation in patients with sick sinus syndrome? Insights from the DANPACE trial. Europace 16: 241-245.

9. Elkayam LU, Koehler JL, Sheldon TJ, Glotzer TV, Rosenthal LS, et al. (2011) The influence of atrial and ventricular pacing on the incidence of atrial fibrillation: a metaanalysis. Pacing Clin Electrophysiol 34: 1593-1599. [Crossref]

10. Svendsen JH, Nielsen JC, Darkner S, Jensen GV, Mortensen LS, et al. (2013) CHADS2 and CHA2DS2-VASc score to assess risk of stroke and death in patients paced for sick sinus syndrome. Heart 99: 843-848. [Crossref]

11. Carlson MD, Ip J, Messenger J, Beau S, Kalbfleisch S, Gervais P, et al. (2003) Atrial Dynamic Overdrive Pacing Trial (ADOPT) Investigators (2003) A new pacemaker algorithm for the treatment of atrial fibrillation: results of the Atrial Dynamic Overdrive Pacing Trial (ADOPT). J Am Coll Cardiol 42: 627-633.

12. Nielsen JC, Kristensen L, Andersen HR, Mortensen PT, Pedersen OL, et al. (2003) A randomized comparison of atrial and dual-chamber pacing in 177 consecutive patients with sick sinus syndrome: echocardiographic and clinical outcome. J Am Coll Cardiol 42: 614-623.

13. Xie JM, Fang F, Zhang Q, Chan JY, Yip GW, et al. (2012) Atrial dysfunction an interatrial dyssynchrony predict atrial high rate episodes: insight into the distinct effects of right atrial appendage pacing. J Cardiovasc Electrophysiol 23: 384-390.

14. Andersen HR, Nielsen JC, Thomsen PE, Thuesen L, Mortensen PT, et al. (1997) Longterm follow-up of patients from a randomised trial of atrial versus ventricular pacing for sick-sinus syndrome. Lancet 350: 1210-1216. [Crossref]

15. Dabrowska-Kugacka A, Lewicka-Nowak E, Rucinski P, Zagozdzon P, Raczak G, et al. (2009) Atrial electromechanical sequence and contraction synchrony during single- and multisite atrial pacing in patients with brady-tachycardia syndrome. Pacing ClinElectrophysiol 32: 591-603.

16. Dabrowska-Kugacka A, Lewicka-Nowak E, Rucinski P, Kozlowski D, Raczak, G, et al. (2010) Single-site Bachmann's bundle pacing is beneficial while coronary sinus pacing results in echocardiographic right heart pacemaker syndrome in brady-tachycardia patients. Circ J 74: 1308-1315.

17. Yu CM, Chan JY, Zhang Q, Omar R, Yip GW, et al. (2009) Biventricular pacing in patients with bradycardia and normal ejection fraction. N Engl J Med 361: 2123-2134. [Crossref]

18. Klein LS, Miles WM, Zipes DP (1990) Effect of atrioventricular interval during pacing or reciprocating tachycardia on atrial size, pressure, and refractory period. Contractionexcitation feedback in human atrium. Circulation 82: 60-68. [Crossref]

Copyright: (C2016 Kiuchi MG. This is an open-access article distributed under the terms of the Creative Commons Attribution License, which permits unrestricted use, distribution, and reproduction in any medium, provided the original author and source are credited. 